\title{
Role of Duplex Ultrasonography in Correlation With CT Angiography In Assessment Of Vascular Complications In Recipients Post Liver Transplantation
}

\author{
${ }^{1}$ Sherif Abu Gamrah, ${ }^{2}$ Marwa Adel Shabaan, ${ }^{1}$ Ahmed Hassan Soliman, ${ }^{1}$ Hala Mohamed Abdel \\ Hamid Badawy
}

Radio-Diagnosis Department, Faculty of Medicine, ${ }^{1}$ Ain Shams University and ${ }^{2}$ Misr University for Science and Technology Correspondence: Hala Mohamed Abdel Hamid Badawy; E-mail: badawyhala@gmail.com ,Mobile: 01118890580

\begin{abstract}
Background: Liver transplantation is currently accepted as a first line treatment for patients with end-stage acute or chronic liver diseases. Graft survival and overall patient survival have steadily improved since the first transplants were performed in the early 1960s, but a significant percentage of transplants develop complications related to vascular and biliary insufficiency. Graft ischemia after liver transplantation is associated with a high incidence of morbidity and mortality. Aim of the Work: to evaluate the sensitivity and specificity of Duplex ultrasound in detection most of vascular complication after liver transplantation in correlation with CTA. Patients and Methods: The study was done from Aug. 2017 until Aug. 2018, where 50-55 cases who underwent living donor liver transplantation with post-operative complications. The cases were done at Ain Shams University Hospital and other private hospitals. All cases where admitted to posttransplanting intensive care unit in the postoperative period where a routine full laboratory and radiological assessment were done. The laboratory assessment included full liver function tests, kidney function tests, complete blood picture, and C-Reactive Protein. Immunosuppressant level and bilirubin level in drains had also done. Results: The patient age ranges from 36 to 53 years with the mean age of 45 years. Most cases have cases had hepatitis $\mathrm{C}$ related cirrhosis $(90 \%)$. One case had veno-occlusive disease related cirrhosis (Budd Chiari syndrome). About $95 \%$ of cases were suffering from end stage liver disease. The results we have reached are that Doppler U/S sensitivity in diagnosis of these complications is $83.8 \%$ and CTA sensitivity is about $100 \%$. Conclusion: It could be concluded that colour duplex is the investigations of choice in postoperative liver transplantation. They can be used as routine steps in the assessment of liver transplant graft postoperatively in recipient patient as early as possible within the first 3 days after operation and used as a late follow up, as they are noninvasive, safe, cheap, and accurate. Routine use of gray-scale US and color duplex showed to minimize the impact of postoperative complications and maximize both graft and recipient patient survival and if any complications suspected, it can be confirmed by CTA
\end{abstract}

Keywords: Duplex Study, CTA, Recipient Patient, Post Liver Transplantation

\section{INTRODUCTION}

Liver transplantation is currently accepted as a first line treatment for patients with end-stage acute or chronic liver diseases ${ }^{(\mathbf{1})}$.

Graft survival and overall patient survival have steadily improved since the first transplants were performed in the early 1960s, but a significant percentage of transplants develop complications related to vascular and biliary insufficiency. Graft ischemia after liver transplantation is associated with a high incidence of morbidity and mortality ${ }^{(2)}$.

Biliary and vascular complications and rejection of the transplanted liver are the main causes of malfunction and loss of the hepatic graft. Advances in medical therapy over the last few years have led to a more efficient diagnosis and treatment of postoperative complications after orthotopic liver transplantation, thereby increasing the survival rate after liver transplantation ${ }^{(3)}$.

Vascular complications include; hepatic artery stenosis and thrombosis, portal vein stenosis and thrombosis, caval and hepatic veins obstruction, arterial pseudo aneurysm. Biliary complications include; biliary leakage, stricture and obstruction ${ }^{(4)}$.

A multimodality approach including ultrasonography and cross-sectional imaging studies often is most effective for diagnosis. Each imaging modality has specific strengths and weaknesses, and the diagnostic usefulness of a modality depends mainly on the patient's characteristics, the clinical purpose of the imaging evaluation, and the expertise of imaging professionals ${ }^{(\mathbf{1})}$.

Ultrasound is the initial imaging modality of choice for detection and follow-up of early and delayed complications from all types of liver transplantation. Knowledge of the surgical technique of liver transplantation permits early detection of complications and prevents misdiagnosis ${ }^{(5)}$.

Duplex US is used to evaluate vessel patancy, and are frequently used to distinguish dilated bile ducts and blood vessels and also can evaluate the extrahepatic portal venous system ${ }^{(6)}$. 
CTA is used in correlation with duplex to confirm any vascular complication (Ref No.).

The aim of this study is to evaluate the sensitivity and specificity of Duplex ultrasound in detection most of vascular complication after liver transplantation in correlation with CTA.

\section{PATIENTS AND METHODS}

This prospective study included a total of 5055 cases with post-operative liver transplantation complications, attending at Ain Shams University Hospital and other private hospitals. Approval of the Ethical Committee and written informed consent from all the subjects were obtained. This study was conducted between Aug 2017 and Aug 2018.

All cases where admitted to post transplanting intensive care unit in the postoperative period where a routine full laboratory and radiological assessment were done. The laboratory assessment included full liver function tests, kidney function tests, complete blood picture, and CReactive Protein. Immunosuppressant level and bilirubin level in drains had also done.

The routine radiological assessment was ultrasound and duplex assessment of the patient as well as chest X-ray; both were done on daily basis. $\mathrm{CT}$ angiography is ordered in the following cases:

Inclusion criteria: Portal vein thrombosis in the ultrasound study. Increased resistive index of hepatic artery. Signs of portal hypertension. Evidence of IVC or hepatic vein thrombosis. Clinical signs of graft failures or rejection (fever, elevated TLC, etc.).

\section{Ultrasound and duplex assessment:}

The ultrasound and duplex assessment were done by GE LOGIC P5 - PHILIPS CLEAR VUE 850 machines using the curved $3.5-5 \mathrm{MHz}$ probe with color and spectral facilities. The liver is first assessed for size and abnormal echogenicity such as area of infarctions or congestions. Assessment of the presence of dilated biliary system was done to exclude biliary strictures, also assessment for any collections and biloma were done and any collection mentioned by site and estimated volume.

\section{Technique of Triphasic CT and CT angiography:}

Cases for abnormal ultrasound Doppler finding or abnormal clinical and laboratory findings were examined by CT either. CT scans were done with a dual 64 channel multi-detector row CT scanner (GE 660 - 64 SLICES).

\section{Image processing and reconstruction:}

All phases of the study were assessed using maximum intensity projections with volume rendering techniques with zooming on areas of abnormal findings.

\section{The statistical methodology:}

The collected data were coded, tabulated, and statistically analyzed using IBM SPSS statistics (Statistical Package for Social Sciences) software version 18.0, IBM Corp., Chicago, USA, 2009.

Descriptive statistics were done for quantitative data as minimum\& maximum of the range as well as mean \pm SD (standard deviation) for quantitative normally distributed data, while it was done for qualitative data as number and percentage.

Inferential analyses were done for qualitative data using Kappa test for agreement between paired categorical data. The level of significance was taken at $\mathrm{P}$ value $<0.050$ is significant, otherwise is non-significant.

Diagnostic characteristics were calculated as follows: Sensitivity $=$ (True positive test $/$ Total positive golden $) \times 100$. Specificity $=($ True negative test / Total negative golden) $\mathrm{x} 100$. Predictive positive value $=($ True positive test $/$ Total positive test $) \mathrm{x} 100$. Predictive negative value $=($ True negative test $/$ Total negative test $) \times 100$ Diagnostic accuracy $=([$ True positive test + True negative test] / Total cases) x 100. Youden's index $=$ sensitivity + specificity -1

\section{RESULTS}

Table (1): The incidence of each complication.

\begin{tabular}{|l|c|}
\hline \multicolumn{1}{|c|}{ Complication } & Number of cases \\
\hline Portal vein thrombosis & 8 \\
\hline Portal vein narrowing & 8 \\
\hline Hepatic artery stenosis & 19 \\
\hline Hepatic artery thrombosis & 8 \\
\hline hepatic vein thrombosis & 3 \\
\hline IVC thrombosis & 6 \\
\hline Total & 52 \\
\hline
\end{tabular}

Table (2): Diagnostic characteristics of US in diagnosis of hepatic artery thrombosis (CT is golden).

\begin{tabular}{|l|c|c|}
\hline \multicolumn{1}{|c|}{ Characters } & Value & 95\% CI \\
\hline Sensitivity & $87.5 \%$ & $47.3 \%-99.7 \%$ \\
\hline Specificity & $97.6 \%$ & $87.4 \%-99.9 \%$ \\
\hline Diagnostic accuracy (DA) & $96.0 \%$ & $86.3 \%-99.5 \%$ \\
\hline Youden's index & $85.1 \%$ & $61.7 \%-100.0 \%$ \\
\hline Positive Predictive value (PPV) & $87.5 \%$ & $47.3 \%-99.7 \%$ \\
\hline Negative Predictive value (NPV) & $97.6 \%$ & $87.4 \%-99.9 \%$ \\
\hline
\end{tabular}

Table (2) shows that: US had moderate diagnostic characteristics in diagnosis of hepatic artery thrombosis. 
Table (3): Diagnostic characteristics of US in diagnosis of hepatic vein thrombosis (CT is golden).

\begin{tabular}{|l|c|c|}
\hline \multicolumn{1}{|c|}{ Characters } & Value & 95\% CI \\
\hline Sensitivity & $100.0 \%$ & $29.2 \%-100.0 \%$ \\
\hline Specificity & $100.0 \%$ & $92.5 \%-100.0 \%$ \\
\hline Diagnostic accuracy (DA) & $100.0 \%$ & $92.9 \%-100.0 \%$ \\
\hline Youden's index & $100.0 \%$ & $100.0 \%-100.0 \%$ \\
\hline Positive Predictive value (PPV) & $100.0 \%$ & $29.2 \%-100.0 \%$ \\
\hline Negative Predictive value (NPV) & $100.0 \%$ & $92.5 \%-100.0 \%$ \\
\hline
\end{tabular}

Table (3) show that US had perfect diagnostic characteristics in diagnosis of hepatic vein thrombosis.

Table (4): Diagnostic characteristics of US in diagnosis of hepatic vein stenosis (CT is golden).

\begin{tabular}{|l|c|c|}
\hline \multicolumn{1}{|c|}{ Characters } & Value & 95\% CI \\
\hline Sensitivity & $75.0 \%$ & $19.4 \%-99.4 \%$ \\
\hline Specificity & $100.0 \%$ & $92.3 \%-100.0 \%$ \\
\hline Diagnostic accuracy (DA) & $98.0 \%$ & $89.4 \%-99.9 \%$ \\
\hline Youden's index & $75.0 \%$ & $32.6 \%-100.0 \%$ \\
\hline Positive Predictive value (PPV) & $100.0 \%$ & $29.2 \%-100.0 \%$ \\
\hline Negative Predictive value (NPV) & $97.9 \%$ & $88.7 \%-99.9 \%$ \\
\hline
\end{tabular}

Table (4) show that US had moderate diagnostic characteristics in diagnosis of hepatic vein stenosis

Table (5): Diagnostic characteristics of US in diagnosis of portal vein thrombosis (CT is golden).

\begin{tabular}{|l|c|c|}
\hline \multicolumn{1}{|c|}{ Characters } & Value & 95\% CI \\
\hline Sensitivity & $70.0 \%$ & $34.8 \%-93.3 \%$ \\
\hline Specificity & $97.5 \%$ & $86.8 \%-99.9 \%$ \\
\hline Diagnostic accuracy (DA) & $92.0 \%$ & $80.8 \%-97.8 \%$ \\
\hline Youden's index & $67.5 \%$ & $38.7 \%-96.3 \%$ \\
\hline Positive Predictive value (PPV) & $87.5 \%$ & $47.3 \%-99.7 \%$ \\
\hline Negative Predictive value (NPV) & $92.9 \%$ & $80.5 \%-98.5 \%$ \\
\hline
\end{tabular}

Table (5) show that US had low diagnostic characteristics in diagnosis of portal vein thrombosis

Table (6): Diagnostic characteristics of US in diagnosis of portal vein stenosis (CT is golden).

\begin{tabular}{|l|c|c|}
\hline \multicolumn{1}{|c|}{ Characters } & Value & 95\% CI \\
\hline Sensitivity & $77.8 \%$ & $40.0 \%-97.2 \%$ \\
\hline Specificity & $97.6 \%$ & $87.1 \%-99.9 \%$ \\
\hline Diagnostic accuracy (DA) & $94.0 \%$ & $83.5 \%-98.7 \%$ \\
\hline Youden's index & $75.3 \%$ & $47.8 \%-100.0 \%$ \\
\hline Positive Predictive value (PPV) & $87.5 \%$ & $47.3 \%-99.7 \%$ \\
\hline Negative Predictive value (NPV) & $95.2 \%$ & $83.8 \%-99.4 \%$ \\
\hline
\end{tabular}

Table (6)show that US had low diagnostic characteristics in diagnosis of portal vein stenosis

Table (7): Diagnostic characteristics of US in diagnosis of inferior vena cava thrombosis (CT is golden).

\begin{tabular}{|l|c|c|}
\hline \multicolumn{1}{|c|}{ Characters } & Value & 95\% CI \\
\hline Sensitivity & $83.3 \%$ & $35.9 \%-99.6 \%$ \\
\hline Specificity & $97.7 \%$ & $88.0 \%-99.9 \%$ \\
\hline Diagnostic accuracy (DA) & $96.0 \%$ & $86.3 \%-99.5 \%$ \\
\hline Youden's index & $81.1 \%$ & $50.9 \%-100.0 \%$ \\
\hline Positive Predictive value (PPV) & $83.3 \%$ & $35.9 \%-99.6 \%$ \\
\hline Negative Predictive value (NPV) & $97.7 \%$ & $88.0 \%-99.9 \%$ \\
\hline
\end{tabular}

Table (7) show that US had moderate diagnostic characteristics in diagnosis of inferior vena cava thrombosis

From the above numbers we can conclude that Duplex US sensitivity in detecting vascular complications in post liver transplantation patients is $83.8 \%$ and the CTA sensitivity is $100 \%$ with highly positive predictive value and positive likehood ratio which mean that positive findings in duplex study is usually indicative of the presence of complication while the negative findings in duplex study doesn't always mean the absence of complications.

\section{DISCUSSION}

Liver transplantation is lifesaving treatment for patient with liver cell failure. Improvement of preoperative selection, surgical techniques, immunosuppressive therapy, postoperative care and follow up has resulted in increased patient graft survival after the transplantation ${ }^{(7)}$.

Ultrasound is the primary imaging modality in the detection and follow-up of early and delayed complications of LT. First, it can be easily performed at the bedside in the intensive care unit during the early post-transplantation phase; it is accessible and non-invasive; and it avoids the use of ionizing radiation. If performed by expert operators, the results are highly reliable. Ultrasound examination requires greyscale and Doppler evaluation for the assessment of the liver parenchyma, biliary tree and vessels. On greyscale evaluation, the normal LT has a homogeneous echogenicity.Pulsed and colour Doppler examination is performed complementarily to evaluate vessel patency and flow spectra.

CT is a second-line imaging technique that is generally used to confirm or exclude clinical suspicious and/or ultrasound findings. The introduction to clinical practice of multidetector CT (MDCT) has allowed for the acquisition of the whole volume of the abdomen, pelvis and possibly also the thorax in a few seconds with high spatial and temporal resolution, thus enabling the incorporation of both angiographic and parenchymal studies into a single acquisition.

Despite the improvement in the liver transplantation surgical techniques and postoperative management, there are still significant 
and life threatening complications that can lead to graft failure and increased patient morbidity and mortality. Imaging is very important in early diagnosis and management of these complications (9).

The importance of stenosis or thrombosis of the hepatic artery is based on the fact that the intrahepatic biliary epithelium is perfused solely by the hepatic artery. Reduced hepatic artery flow can lead to graft ischemia, affecting at first the bile ducts, and eventually graft failure. We analyzed our study group on the basis of derived thresholds of RI less than 0.5 and SAT greater than $0.08 \mathrm{~s}$ for identification of marked HAS or HAT. Requiring both RI and SAT to be abnormal to predict HAS or HAT, Doppler US resulted in a diagnostic sensitivity and a specificity of $87.5-100 \%$ and 96.9 $97.6 \%$, respectively and considering the CT is the gold standard it has a sensitivity of $47.3 \%-99.7 \%$ for HAS and has a sensitivity of $81.5 \%-100.0 \%$ in detecting HAT.

Our results are superior to Dodd et al. ${ }^{(2)}$ who reported a $73 \%$ sensitivity and specificity for the detection of marked hepatic arterial disease by using the same parameters. In another study that was consisted of 46 patients, Platt et al. ${ }^{(15)}$ reported a sensitivity of $67 \%$ and a specificity of $96 \%$ for Doppler US in detecting HAS by using similar parameters and a sensitivity of $100 \%$ for CTA.

Thrombosis and/or stenosis of the portal vein and hepatic veins are reported to occur in 1$12.5 \%$ of liver recipients.We observed portal vein complications in $12-18 \%$, and hepatic vein complications in $3 \%$ of liver transplantations. For portal vein stenosis, we prospectively analyzed our study group on the basis of Doppler US criteria those previously reported by Dodd et al. ${ }^{(2)}$. Our suggested thresholds for marked portal vein stenosis are consisted of a visualized portal vein diameter of $2.5 \mathrm{~mm}$ or less detected on sonography, and an accelerated flow at the stricture or a poststenotic jet at least five times of the recordings in the proximal portal vein revealed by spectral Doppler US imaging. We diagnosed five instances of marked portal vein stenosis including both of the diagnostic parameters described above and confirmed by portal CT venography. Combined use of sonography, spectral Doppler US parameters and CT venography resulted in a diagnostic sensitivity and specificity of $100 \%$ for marked portal vein stenosis/thrombosis. Our data reported
$70-77.8 \%$ and $97.6 \%$ sensitivity and specificity respectively for doppler US and 93\% and 97\% sensitivity and specificity for CT.

Hepatic vein stenosis has emerged as one of the important vascular complications of living donor liver transplantation. In our study, the incidence of hepatic vein stenosis occurred in $6 \%$ of living donor liver recipients. In our study, all of the three cases of proven hepatic vein stenosis showed a persistent monophasic wave pattern on Doppler US examinations.

We concluded that duplex study has a sensitivity of $83.3 \%$ in detecting IVC thrombosis.and considering the CT is the gold standard it has a sensitivity of 35.9\%99.6\% in detecting IVC thrombosis.

We recognize that our study has some limitations and biases. Limitations of our study mainly related to the prospective nature of the study, low incidence of occurrence of some vascular complications like pseudoaneurysm and splenic artery syndrome as well as low sample volume.

\section{CONCLUSION}

Duplex US are the investigations of choice in postoperative liver transplantation, they can be used as a routine steps in the assessment of liver transplant graft postoperatively in recipient patient as early as possible within the first 3 days after operation and used as a late follow up, as they are noninvasive, safe, cheap, and accurate. Routine use of gray-scale US and color Doppler showed to minimize the impact of postoperative complications and maximize both graft and recipient patient survival.

\section{REFERENCES}

1- Angela C, Roberto B, Marcelino A et al. (2007): Complications of liver transplantation. Multimodality imaging approach. Radiographics, 27:1401

2- Dodd GD, Memel DS, Zajko AB et al. (2001) Hepatic artery stenosis and thrombosis in transplant recipients: Doppler diagnosis with resistive index and systolic acceleration time. Radiology, 192:657-661

3- Hong SS, Ah YK, Hye JK et al. (2006): Inferior phrenic arterial bleeding after adult liver transplantation, AJR; 187:15. 
4- Horrow M, Beth MB, David JR, Cosme M (2007): Sonography of hepatic artery thrombosis in transplant recipients. https:// www. ajronline. org/ doi/ abs/ 10. 2214/ AJR.07.2217

5- Quiroga S, Sebastià MC, Margarit C, Castells L, Rosa Boyé $R$ and Castells AA (2001): Complications of Orthotopic Liver Transplantation: Spectrum of Findings with Helical CT. Radiographics, 21: 1085-1102.

6- Jane D, Muradali D and Wilson SR (2003): U.S of liver transplantation. Noraml and Abnormal Radiographic; 28:1093-1114.

7- Jonathan BK, Newman PA, Sammons LG and Kane RA (2004): Optimizing Doppler and color flow US: application to hepatic sonography. RadioGraphics, 24:657.

8- Busuttil RW and Lake JR (2004): Role of tacrolimus in the evolution of liver transplantation. Transplantation,77(9): S44-51.

9- Erbay N, Raptopoulos V, Pomfret EA, Kamel IR, Kruskal JB (2003): Living donor liver transplantation in adults: vascular variants important in surgical planning for donors and recipients. American Journal of Roentgenology, 181(1):109-14.

10- Hussain HK and Nghiem HV (2002): Imaging of hepatic transplantation. Clinics in Liver Disease, 6(1):247-70.
11- Lomas DJ, Britton PD, Farman P, Coulden R, Egan A, Jamieson GN, Revell S, Johnston $P$ (1992): Duplex Doppler ultrasound for the detection of vascular occlusion following liver transplantation in children. Clinical Radiology, 46(1):38-42.

12- Lukšaitè R, Samuilis A, Sokolovas V, Tamošiūnas AE, Strupas K (2012): Ultrasound imaging of vascular complications after adult orthotopic liver transplantation, 66(2): 113-115.

13- Nolten A and Sproat IA (1996): Hepatic artery thrombosis after liver transplantation: temporal accuracy of diagnosis with duplex US and the syndrome of impending thrombosis. Radiology, 198:553-559.

\section{4- Bhargava P, Vaidya S, Dick AA, Dighe M} (2011): Imaging of orthotopic liver transplantation. American Journal of Roentgenology,196(3):WS15-25.

15- Platt JF, Yutzy GG, Bude RO, Ellis JH, Rubin JM(2012): Use of Doppler sonography for revealing hepatic artery stenosis in liver transplant recipients. AJR.,168:473-476. 